\title{
Solid neuroendocrine breast carcinomas: Incidence, clinico-pathological features and immunohistochemical profiling
}

\author{
EUGENI LÓPEZ-BONET ${ }^{1}$, MIGUEL ALONSO-RUANO ${ }^{2}$, GRACIELA BARRAZA ${ }^{1}$, \\ ALEJANDRO VAZQUEZ-MARTIN ${ }^{3,4}$, LUIS BERNADÓ ${ }^{1}$ and JAVIER A. MENENDEZ $Z^{3,4}$
}

\begin{abstract}
Departments of ${ }^{1}$ Pathology, and ${ }^{2}$ Gynaecology, Dr Josep Trueta University Hospital of Girona, Girona, Catalonia, ${ }^{3}$ Catalan Institute of Oncology (ICO), Girona; ${ }^{4}$ Girona Biomedical Research Institute (IdIBGi), Girona, Catalonia, Spain
\end{abstract}

Received June 11, 2008; Accepted September 8, 2008

DOI: 10.3892/or_00000154

\begin{abstract}
Primary pure neuroendocrine breast carcinomas (NEBC) have been considered special features within conventional breast carcinomas until recently. Indeed, the actual incidence of NEBC in BC populations has remained largely unknown due to the lack of unambiguous diagnostic criteria. In 2003, the World Health Organization (WHO) classification of breast tumors definitely established that the immunohistochemical expression of NE markers in more than $50 \%$ of the tumor cell population is the unique requisite for NEBC diagnosis. Herein, we sought to determine the incidence, the clinico-pathological features and the immunohistochemical profile of NEBC in a large series of 1368 infiltrating breast tumors collected from 1989 to 2008 in our institution (Dr Josep Trueta University Hospital, Girona, Catalonia). Twelve cases were initially selected to fulfil histopathological patterns compatible with NEBC. Clinical data along with histological and immunohistochemical profiles were collected in all cases. The criterion inclusion was the presence of more than $50 \%$ tumor immunoreactivity for one of NE markers including chromogranin, synaptophysin and CD56. Only 7 tumors fully satisfied the NEBC criteria established by the WHO (0.5\% prevalence). All the NECB were grade 2 ductal carcinoma infiltrating (DCI) with tumor sizes ranging from 7 to $55 \mathrm{~mm}$. Lymphovascular tumoral emboli was present in 4 cases $(57.1 \%$ of NEBC) and mucinous features occurred in 2 cases (28.5\% of NEBC). Axillary lymph nodes were metastatic in 3 cases (42.8\% of NEBC). A positive status for estrogen receptor (ER), progesterone receptor (PR) and synaptophysin was observed in 7 cases ( $100 \%$ of NEBC). None of the NEBC displayed HER2 overexpression. All the patients bearing NECB received hormone
\end{abstract}

Correspondence to: Dr Javier A. Menendez, Catalan Institute of Oncology-Girona (ICO Girona), Dr Josep Trueta University Hospital of Girona Avenida de Francia s/n; E-17007 Girona, Catalonia, Spain

E-mail: jmenendez@ico.scs.es; jmenendez@iconcologia.net

Key words: neuroendocrine, breast cancer subtypes, luminal therapy and 4 of them underwent radiotherapy and/or chemotherapy. Of note, none of the NEBC patients died from BC-related causes after a median follow-up of 51 months. These findings revealed that: a) Pure solid NEBC do not significantly differ from other breast carcinomas in terms of general clinical features; b) NEBC do not exhibit an aggressive behavior despite the presence of adverse prognostic factors; and c) NEBC immunohistochemical profile mainly corresponds to that of the Luminal A BC subtype. Although it remains to be elucidated whether the good prognosis of NEBC relates to the intrinsic nature of the tumor and/or to a high rate of treatment responses, their immunohistochemical profile strongly suggest that NEBC belong to the Luminal A BC subtype. Forthcoming studies should definitely determine if the clinico-pathological features of NEBC indeed represent an independent good-prognosis subgroup of $\mathrm{BC}$ gene signature.

\section{Introduction}

The World Health Organization (WHO) classification of breast tumors has recently clarified the confusing interpretation of the phenomenon of neuroendocrine (NE) differentiation in breast cancer disease. WHO's classification clearly establish that the immunohistochemical expression of NE markers in more than $50 \%$ of the tumor cell population is the unique requisite for the diagnosis of primary pure neuroendocrine breast carcinomas (NEBC) (1).

In 1977 the first eight cases of breast tumors were published classified as NE by the presence of argyrophilia and cytoplasmic dense core granules (2). In 1989, Papotti et al (3) reported that about $8 \%$ of breast tumors displayed some degree of NE differentiation when they analyzed a consecutive series of 100 infiltrating breast carcinomas (3). However, the actual incidence of pure-NE-differentiated breast tumors was less than $1 \%$. In this regard, it is well known that some NE differentiation can be identified in subsets of breast carcinomas as scattered cells. Yet, the prevalence of pure NEBC when following strictly WHO criteria remains to be established (1).

Here, we sought to determine the prevalence of NEBC in our institution (Dr Josep Trueta University Hospital, Girona, Catalonia) using a large series of 1368 breast infiltrative tumors collected from 1989 to 2008. In addition, we evaluated 
Table I. Immunohistochemical methodology.

\begin{tabular}{lllcc}
\hline Antibody & \multicolumn{1}{c}{ Clone } & \multicolumn{1}{c}{ Dilution } & Antigenic retrieval & Commercial source \\
\hline ER & $6 \mathrm{~F} 11$ & $1: 50$ & 1 & Novocastra \\
PR & 116 & $1: 100$ & 1 & Novocastra \\
HER2 & & HERCEPTEST & & Dako \\
Ki67 & MM1 & $1: 50$ & 1 & Novocastra \\
p53 & DO-7 & $1: 400$ & 1 & Dako \\
Chromogranin & Polyclonal & Not diluted & No & Dako \\
Synaptophysin & Polyclonal & $1: 1$ & 1 & Dako \\
CD56 & $123 C 3$ & $1: 100$ & 1 & Zymed \\
\hline
\end{tabular}

1, EDTA buffer; pH 8.0 (pressure cooker $3 \mathrm{~min}$ ).

Table II. Clinical findings in patients with NEBC.

\begin{tabular}{lcllccr}
\hline Case & Age & Surgery & Breast localization & Multiplicity & Tumor size (mm) & Node axillary status $^{\mathrm{a}}$ \\
\hline 1 & 58 & Mastectomy & Retroareolar, right & Yes & 55 & $14 / 26$ \\
2 & 35 & Mastectomy & Upper-internal, right & No & 23 & $0 / 21$ \\
3 & 52 & Mastectomy & Retroareolar, right & No & 45 & $16 / 18$ \\
4 & 64 & Trucut biopsy & Upper-external, right & Yes & 50 & Not done \\
5 & 88 & Mastectomy & Upper-external, right & No & 41 & $1 / 10$ \\
6 & 69 & Conservative & Inferior-external, right & No & 7 & $0 / 13$ \\
7 & 63 & Mastectomy & Upper-external, left & No & 22 & $0 / 10$ \\
\hline
\end{tabular}

${ }^{a}$ Number of metastatic nodes/total number of nodes isolated.

both the clinico-pathological features and the immunohistochemical profile of NEBC in order to reveal histopathological patterns and/or prognostic factors dissimilar from those of conventional breast carcinomas.

\section{Materials and methods}

Breast cancer specimens. We revised the entire archive of our breast cancer collection in the Department of Pathology of the Dr Josep Trueta University Hospital (Girona, Catalonia) from 1989 until 2008. We first selected all the cases with any histological feature of NE differentiation. Hematoxylin/eosin sections and immunohistochemistry studies were repeated in some ancient cases to avoid dyeing deficiencies. Immunohistochemical markers included synapthophisin, chromogranin, CD56, estrogen receptor (ER), progesterone receptor (PR), HER2 (erbB-2), p53 and Ki67 (Table I). ER and PR were considered positive if $>1 \%$ of nuclear invasive carcinoma cell staining was observed. p53 and Ki67 were scored according to the percentage of nuclear staining in tumor cells. p53 score was divided into 5 categories $(0,1-25 \%, 26-50 \%, 51-75 \%$, $>75 \%$ ) whereas Ki67 was evaluated in a continuous scale. The criterion inclusion was the presence of $>50 \%$ of invasive tumor cells with cytoplasmic immunoreaction for synaptho- phisin, chromogranin or CD56. Histopathological interpretation was performed by two different observers in a double blind manner.

\section{Results}

Incidence of $N E B C$. From the entire series of 1368 breast infiltrating breast carcinomas, 12 cases were initially selected to fulfil histopathological patterns compatible with NEBC. Finally, only 7 tumors fully satisfied the NEBC criteria established by the WHO (i.e. the presence of $>50 \%$ tumor immunoreactivity for one of NE markers including chromogranin, synaptophysin and CD56). Therefore, the incidence of NEBC in this large series of breast carcinomas was as low as $0.5 \%$.

Clinical features of NEBC. Clinical data from NEBC patients are listed in Table II. The age of NEBC patients ranged from 35 to 88 (median 63). Surgical treatment was performed in 6 patients. Radical mastectomy in $5 / 6$ cases $(83.3 \%)$ and conservative surgery in $1 / 6(16.6 \%)$. The remaining case debuted with metastasis in the soft tissue of the cheek and solely a needle-core (i.e. Trucut) biopsy was available. A retroareolar localization of the tumor was identified in two 
Table III. Pathological findings in patients with NEBC.

\begin{tabular}{lccccc}
\hline Case & Histological type & Histological grade & Lymphovascular tumor emboli & Mucinous features & DCIS $>20 \%$ \\
\hline 1 & DCI & 2 & Yes & No & No \\
2 & DCI & 2 & Yes & Yes & No \\
3 & DCI & 2 & Yes & No & No \\
4 & DCI & 2 & No & No & No \\
5 & DCI & 2 & No & No & No \\
6 & DCI & 2 & No & Yes & No \\
7 & DCI & 2 & Yes
\end{tabular}

aScarff-Bloom-Richardson combinated histological grade. DCI, ductal carcinoma infiltrating; DCIS, ductal carcinoma 'in situ' .

Table IV. Immunohistochemical profiling of NEBC.

\begin{tabular}{lcccccccc}
\hline Case & ER & PR & HER2 & p53 $(\%)$ & Ki67 $(\%)$ & Chromogranin $(>50 \%)$ & Synaptophysin $(>50 \%)$ & CD56 $(>50 \%)$ \\
\hline 1 & Positive & Positive & $1+$ & 0 & 10 & No $^{\mathrm{a}}$ & Yes & No \\
2 & Positive & Positive & $2+$ & 0 & 9 & $\mathrm{No}^{\mathrm{a}}$ & Yes & No \\
3 & Positive & Positive & 0 & 0 & 37 & $\mathrm{No}^{\mathrm{a}}$ & Yes & No \\
4 & Positive & Positive & 0 & $50-75$ & 31 & No & Yes & No \\
5 & Positive & Positive & 0 & 0 & 8 & No $^{\mathrm{a}}$ & Yes & No \\
6 & Positive & Positive & 0 & $1-25$ & 7 & $\mathrm{No}^{\mathrm{a}}$ & Yes & No \\
7 & Positive & Positive & 0 & $1-25$ & 25 & & & \\
\hline
\end{tabular}

${ }^{\mathrm{a}}$ Cases with only focal immunostaining in $<50 \%$ of the tumor cells.

cases. Multiplicity was present in 1 case. Tumor size ranged from 7 to $55 \mathrm{~mm}$ (mean 34.7; median 41). Radical dissection of axillary nodes but not selective dissection of sentinel node was performed in 6 cases. The number of dissected axillary lymph nodes oscillated from 10 to 26 (mean 16.3; median 15.5) with no metastasis in 3 cases, one node metastasis in one case and more than $3 / 4$ metastatic nodes in the two remaining cases (14 and 16 metastatic nodes, respectively).

Pathological features of $N E B C$. Pathological findings from NEBC patients are listed in Table III. The tumor type in all the NEBC cases in our series was ductal infiltrating carcinoma (DCI). When the Scarff-Bloom-Richardson (SBR) system was used to assign a grade to a tumor all the NEBC were classified as grade 2 (moderately differentiated). Mucinous differentiation occurred in 2/7 cases (29\%).

Immunohistochemical profiling of NEBC. Immunohistochemical profiles of NEBC patients are listed in Table IV, $7 / 7$ cases $(100 \%)$ were positive for ER and PR immunoreactivity. All the 7 selected NEBC cases (100\%) were positive for synapthophysin or chromogranin in $>50 \%$ of tumor cells (Fig. 1a). Metastatic nodes displayed histological features similar to those found in the primary tumor (Fig. 1b). In one case it was possible to perform an ultrastructural study clearly revealing the occurrence of double membrane-bound dense-core granules and vesicles (Fig. 1c).

Treatment and prognosis of NEBC patients. Treatment schedules and follow-up of NEBC patients are listed in Table V, 7/7 cases (100\%) received hormonotherapy with tamoxifen. Two patients received adjuvant therapy (5-fluorouracil, epirubicin and cyclophosphamide -FEC- and FEC $\rightarrow$ taxane -docetaxel-) $\rightarrow$ capecitabine in one case with metastatic disease). Neoadjuvant treatments with hormonotherapy (i.e. letrozole) and chemotherapy (i.e. FEC) were performed in two patients. Five patients bearing NEBC were alive (death of the two remaining cases was due to non-breast cancer causes) in the last clinical control with a mean follow-up of 51 months (range 3-84). Remarkably, the patient that debuted with metastasis in the soft tissue of the cheek prior to diagnosis of NEBC was still alive 7 years later.

\section{Discussion}

$\mathrm{NE}$ features have been recognized for many years in human breast tumors. However, the actual prevalence of NE breast lesions has been difficult to establish mainly due to the confusing diagnostic criteria. Although some efforts were made to study breast carcinomas displaying NE features 

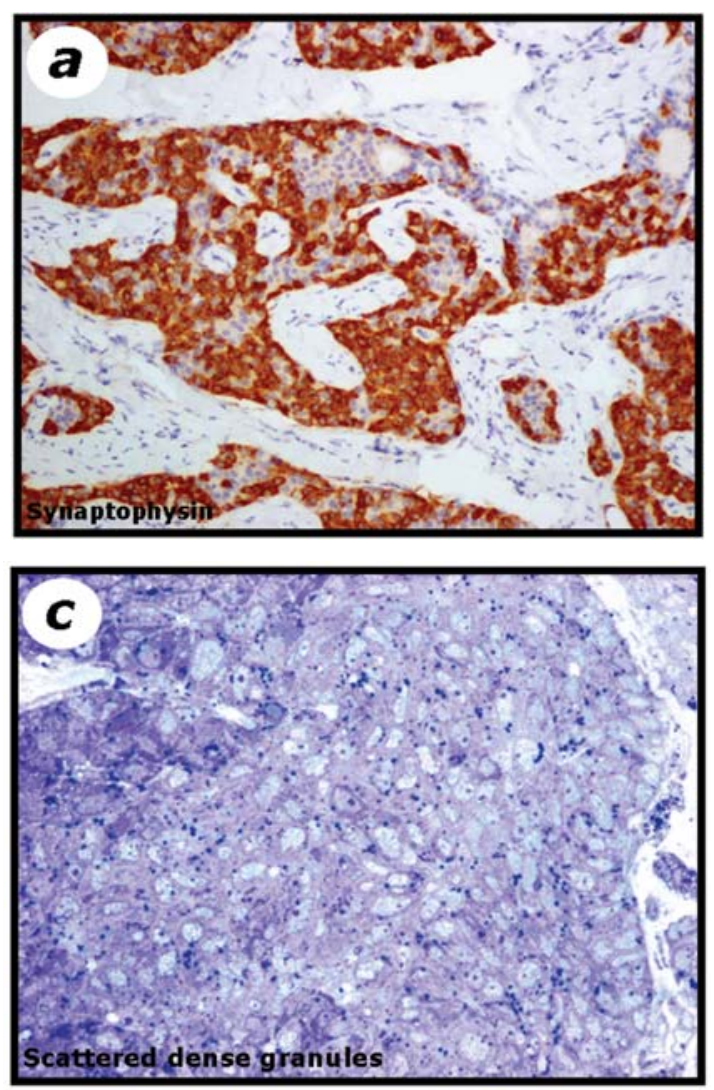

based on morphological features and immunohistochemical markers $(3,6)$, NEBC were not recognized as single BC entities until the last WHO's classification of breast carcinomas in $2003(4,5)$. Using a large series of 1368 infiltrating breast tumors we observed a prevalence of NEBC as low as $0.5 \%$. This level of NEBC incidence does not significantly differ from that reported in earlier studies. Thus, a retrospective review of the mammograms of 1845 histopathologically proven breast cancer cases revealed five NEBC $(0.3 \%)(7)$.

The unique requisite to diagnose a NEBC is the immunohistochemical expression of NE markers in $>50 \%$ of the tumor cell population (1). Although it is widely accepted that

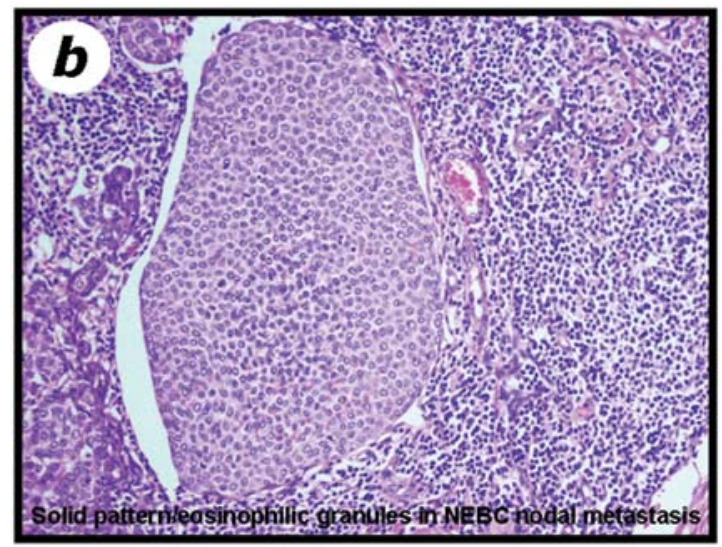

Figure 1. NEBC immunohistochemical features. (a) All the 7 selected NEBC in our study $(100 \%)$ were positive for synapthophysin or chromogranin in $>50 \%$ of tumor cells. Figure shows strong cytoplasmic immunoreactivity for synaptophysin (case 6 in Tables II to V). (b) Shows nodal metastases in which both the solid pattern is preserved and the eosinophilic granules are still present in a ganglionar marginal sinus tumoral permeation (case 1 in Tables II to V). (c) Semifine section of cheek soft tissue metastases showing scattered dense granules in the cytoplasm of tumoral cells (case 4 in Tables II to V).

immunohistochemical demonstration of chromogranin or synapthophysin correlates well with the ultrastructural demonstration of dense-core granules $(3,8)$ it could be argued that this cut-off is conceptually arbitrary and, indeed, many of the NEBC share morphological features regardless their NE immunohistochemical profile. Therefore, it appears that novel markers are urgently needed to better define this BC subgroup as the expression of multiple NE-related genes may encode for a wide spectrum of NEBC traits. For instance, the expression of NE markers is inconsistent in the very rare small cell (oar cell) NE carcinoma of the breast and the prognosis in these patients may not be as poor as previously suggested $(9,10)$. The remaining group (i.e. solid NEBC) clearly displays NE histological features. A major NEBC feature relates to the disposition of tumor cells in solid nests with a tendency toward peripheral palisading, which constitutes a reminiscence of either in situ or infiltrating lobular carcinomas (Fig. 2a-c). This feature occurred in all the seven

Table V. Treatment schedules and follow-up in patients with NEBC.

\begin{tabular}{|c|c|c|c|c|c|c|}
\hline Case & Radiotherapy & Hormonotherapy & $\begin{array}{c}\text { Neoadjuvant } \\
\text { treatment }\end{array}$ & $\begin{array}{c}\text { Adjuvant } \\
\text { chemotherapy }\end{array}$ & Follow-up (months) & Disease state \\
\hline 1 & n.a. & Yes & Letrozole & n.a. & 3.2 & Alive, free of disease \\
\hline 2 & Yes & Yes & FEC & No & 2.7 & Alive, free of disease \\
\hline 3 & Yes & Yes & No & FEC & 58.6 & Alive, free of disease \\
\hline 4 & No & Yes & No & FEC-T-CP & 84.3 & Alive, metastatic disease \\
\hline 5 & No & Yes & No & No & 34 & Dead, Non-breast related \\
\hline 6 & No & Yes & No & No & 63.2 & Dead, Non-breast related \\
\hline 7 & No & Yes & No & No & 115.5 & Alive, free of disease \\
\hline
\end{tabular}

n.a., data not available. FEC, 5-fluoruracil, epirubicin and cyclophosphamide; T, taxane (docetaxel); CP, capecitabine. 

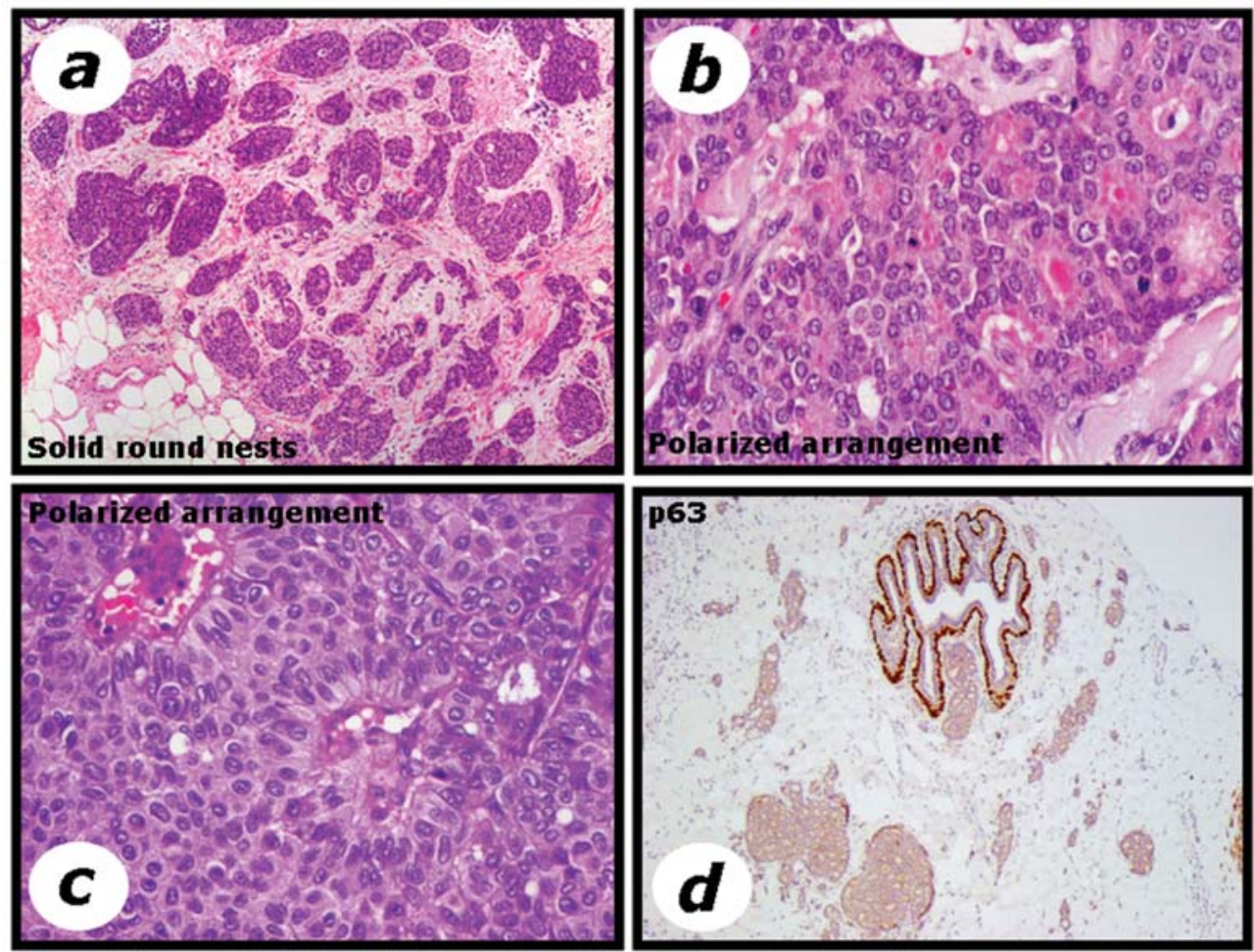

Figure 2. Major histological features in NEBC. (a) NEBC usually display a disposition of solid round nests that infiltrate extensively the surrounding stoma (case 6 in Tables II to V). (b) Shows a polarized arrangement of tumor cells around lumina which resembles rosette-like structures with eosinophilic granules (case 6 in Tables II to V). (c) Shows a polarized arrangement that is around transversal papilla (case 5 in Tables II to V). (d) p63 nuclear immunostaining of the marginal myoepithelial layer in a non-neoplastic breast duct markedly contrasts with the negativity of the peripheral palisading in the tumoral solid nests (case 1 in Tables II to V).

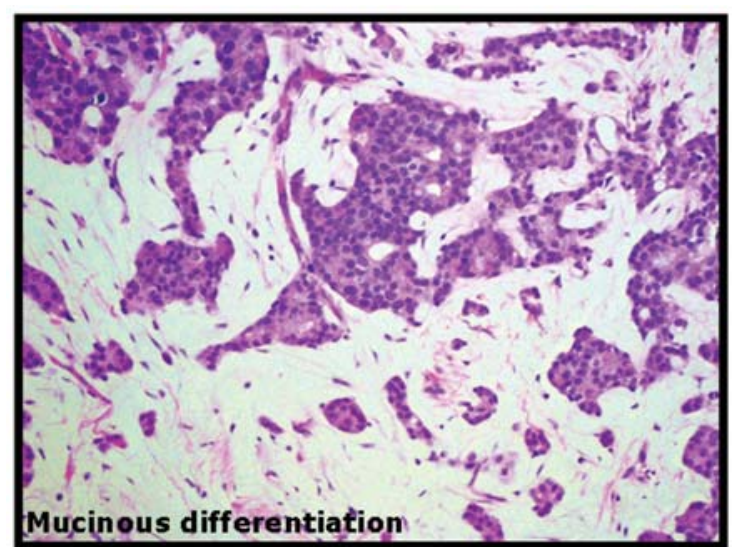

Figure 3. Mucinous differentiation in NEBC. A remarkable histological feature observed in NEBC is the incidence of mucinous differentiation. In the figure, islands of tumor cells appear to float in a loose mucous-like stroma. This histological feature displays a focal nature in NEBC and, therefore, is not enough to be considered a mucinous carcinoma itself (case 1 in Tables II to V).

cases of NEBC in our series. In addition, we occasionally observed polarized arrangements of tumor cells displaying eosinophilic granules around lumina. Altogether, these histological features model rosette-like structures in a classical carcinoid-like pattern along with a cordonal arrangement of the infiltrating tumor cells. Although the solid nests findings alone might be confusing to the diagnosis of NEBC as they can also be found in in situ/infiltrating lobular and intraductal carcinomas both can be excluded when considering E-cadherin positivity and p63 negativity of the palisading cells, respectively (Fig. 2d). Indeed, many of the published NEBC displaying intraductal carcinoma features could be better discriminated using myoepithelial markers. Nevertheless, the ultimate diagnosis of NEBC is given by the presence of synapthophysin or chromogranin immunohistochemical expression in more than $50 \%$ of the BC cell population. Both markers were found in our study. However, synapthophysin strongly stained in all the seven cases whereas chromogranin staining stained with a focal distribution in five tumors. These findings may relate to the fact that most diagnostic laboratory employ monoclonal antibodies raised against chromogranin A. It is obvious that if some NEBC contain chromogranin $\mathrm{B}$ they will be scored as negative for chromogranin when using an antibody that exclusively recognizes the isoform A of chromogranin. Also, the fact that all the NEBC cases were negative for CD56 likely relates to the tendency of the anti-CD56 monoclonal antibody to preferentially immunoreact against undifferentiated small cell carcinomas but not against more differentiated NE carcinomas. Synaptophysin represents the major protein of the synaptic vesicle and is widely expressed in neurons but it is commonly present in other NE tissues and in their corresponding tumors. Another remarkable histological feature observed in two of our seven NEBC is the occurrence of mucinous differentiation (Fig. 3). Although the focal amount of mucinous differentiation in NEBC cannot be considered a mucinous carcinoma itself 
it might correlate with the good prognosis of NE carcinomas (11-13). In agreement with earlier studies, all the NECB in our series were classified as grade 2 (moderately differentiated) when the histological grade was classified according to modified Scarff-Bloom-Richardson histological grading criteria (14). No other specific histological features were capable to discriminate NEBC from other types of infiltrating BC.

All the NEBC cases remained alive after a mean followup of 51 months. Of the three cases with node axillary metastases at the time of diagnosis, two remained free of tumor disease after a follow-up of 9.5 and $~ 3$ years, respectively. In this regard, prognostic factors in NEBC do not differ from those classically considered in other BC subtypes $(11,15,16)$. Tumor size, nodal status, histological grade, lymphatic tumor emboli, small cell NE subtype, mucinous differentiation, hormonal receptor and HER2 status are reliable features indicative of the clinical outcome and treatment responses in BC. However, the limited number of NEBC cases in our and other series does not allow establishing significant correlations with specific prognostic factors. It could be speculated that NEBC do not present specific clinical and/or imaging features that separate them from other $B C$ types (17). It has been reported that NEBC presentation is accompanied by fairly-well circumscribed, dense round or irregular masses with speculated or lobulated margins and homogenous enhancement with a time-intensity curve localized in the subareolar region and associated with bloodstained discharge from the nipple (7). None of the seven NEBC cases in our series debuted with nipple blood discharge but two of them were localized in the retroareolar region.

Regardless of both the nomenclature and the classification of NE tumors it should be noted that specific biological behaviors are, at least in part, site-dependent. Therefore, it would be relevant to definitely describe both the type and the malignant potential of NE carcinomas. In localizations other than breast, prognostic parameters of NE tumors are based on tumor size and site, the presence of local invasion, angioinvasion and metastasis. Cytological atypia, mitotic index and proliferating rate as assessed by Ki67 staining are also important prognostic criteria in NE carcinomas. However, all these prognostic factors are also valid in other BC. Moreover, all the NEBC described in our study showed a positive status for estrogen and progesterone receptors while only one case exhibited HER2 overexpression (2+). If we extrapolate both the clinico-pathological features and the immunohistochemical profiling of NECB in our series it is reasonable to suggest that they likely belong to the Luminal A sub-type of breast carcinomas. Forthcoming studies should definitely determine if the clinico-pathological features of NEBC indeed represent an independent good-prognosis subgroup within the $\mathrm{BC}$ gene signature.

\section{Acknowledgments}

Javier A. Menendez is the recipient of a Basic, Clinical and Translational Research Award (BCTR0600894) from the Susan G. Komen Breast Cancer Foundation (TX, USA). This work was supported in part by Instituto de Salud Carlos III (Ministerio de Sanidad y Consumo, Fondo de Investigación Sanitaria-FIS-, Spain, Grants CP05-00090 and PI06-0778 to Javier A. Menendez, and Grant RD06-0020-0028 to Javier A. Menendez). Javier A. Menendez was also supported by a Grant from the Fundación Científica de la Asociación Española Contra el Cáncer (AECC, Spain).

\section{References}

1. Tavassoli FA and Devilee P (eds). World Health Organization classification of tumours. In: Tumours of the Breast and Female Genital Organs. IARC Press, Lyon, 2003.

2. Cubilla AL and Woodruff JM: Primary carcinoid tumor of the breast: a case report of eight patients. Am J Surg Pathol 1: 283-292, 1997.

3. Papotti M, Macrì L, Finzi G, Capella C, Eusebi V and Bussolati G: Neuroendocrine differentiation in carcinomas of the breast: a study of 51 cases. Semin Diagn Pathol 6: 174-188, 1989.

4. Rosen PP: Rosen's Breast Pathology. Lippincott-Raven, PA, 1997.

5. Sapino A, Righi L, Cassoni P, Papotti M, Pietribiasi F and Bussolati G: Expression of the neuroendocrine phenotype in carcinomas of the breast. Semin Diagn Pathol 17: 127-137, 2000

6. Scopsi L, Andreola S, Pilotti S, et al: Argyrophilia and granin (chromogranin/secretogranin) expression in female breast carcinomas. Their relationship to survival and other disease parameters. Am J Surg Pathol 16: 561-576, 1992.

7. Günhan-Bilgen I, Zekioglu O, Ustün EE, Memis A and Erhan Y: Neuroendocrine differentiated breast carcinoma: imaging features correlated with clinical and histopathological findings. Eur Radiol 13: 788-793, 2003.

8. Capella C, Usellini L, Papotti M, Macrì L, Finzi G, Eusebi V and Bussolati G: Ultrastructural features of neuroendocrine differentiated carcinomas of the breast. Ultrastruct Pathol 14: 321-334, 1990.

9. Adegbola T, Connolly CE and Mortimer G: Small cell neuroendocrine carcinoma of the breast: a report of three cases and review of the literature. J Clin Pathol 58: 775-778, 2005.

10. Shin SJ, DeLellis RA, Ying L and Rosen PP: Small cell carcinoma of the breast: a clinicopathologic and immunohistochemical study of nine patients. Am J Surg Pathol 24: 1231-1238, 2000 .

11. Sapino A, Papotti M, Righi L, Cassoni P, Chiusa L and Bussolati G: Clinical significance of neuroendocrine carcinoma of the breast. Ann Oncol 12: 115-117, 2001

12. Scopsi L, Andreola S, Pilotti S, Bufalino R, Baldini MT, Testori A and Rilke F: Mucinous carcinoma of the breast. A clinicopathologic, histochemical, and immunocytochemical study with special reference to neuroendocrine differentiation. Am J Surg Pathol 18: 702-711, 1994.

13. Tse GM, Ma TK, Chu WC, Lam WW, Poon CS and Chan WC: Neuroendocrine differentiation in pure type mammary mucinous carcinoma is associated with favorable histologic and immunohistochemical parameters. Mod Pathol 17: 568-572, 2004.

14. Zekioglu O, Erhan Y, Ciris M and Bayramoglu H: Neuroendocrine differentiated carcinomas of the breast: a distinct entity. Breast 12: 251-257, 2003.

15. Makretsov N, Gilks CB, Coldman AJ, Hayes M and Huntsman D Tissue microarray analysis of neuroendocrine differentiation and its prognostic significance in breast cancer. Hum Pathol 34: 1001-1008, 2003

16. Miremadi A, Pinder SE, Lee AH, Bell JA, Paish EC, Wencyk P, Elston CW, Nicholson RI, Blamey RW, Robertson JF and Ellis IO: Neuroendocrine differentiation and prognosis in breast adenocarcinoma. Histopathology 40: 215-222, 2002.

17. Fujimoto Y, Yagyu R, Murase K, Kawajiri H, Ohtani H, Arimoto Y, Yamamura T, Inoue T and Moritani S: A case of solid neuroendocrine carcinoma of the breast in a 40 -year-old woman. Breast Cancer 14: 250-253, 2007. 